\title{
Estimation of temporal gait parameters of multiple sclerosis patients in clinical setting using inertial sensors
}

\author{
Julius Griškevičius ${ }^{1}$, Vigita Apanskienė ${ }^{2}$ Jurgita Žižiene் $\dot{e}^{3}$, Kristina Daunoravičienė ${ }^{4}$, \\ Agnė Ovčinikova ${ }^{5}$, Rasa Kizlaitiene் ${ }^{6}$, Ieva Sereike் ${ }^{7}$, Gintaras Kaubrys ${ }^{8}$, Jolanta Pauk ${ }^{9}$, \\ Adam Idźkowski ${ }^{10}$ \\ $1,2,3,4$ Vilnius Gediminas Technical University, Lithuania \\ $5,6,7,8$ Clinics of Neurology and Neurosurgery of Vilnius University Hospital Santariškių Klinikos, \\ Lithuania \\ 9, 10 Bialystok University of Technology, Poland \\ E-mails: ${ }^{1}$ julius.griskevicius@vgtu.lt (corresponding author), ${ }^{2}$ vigita.apanskiene@stud.vgtu.lt, \\ ${ }^{3}$ jurgita.ziziene@vgtu.lt ${ }^{4}$ kristina.daunoraviciene@vgtu.lt,${ }^{5}$ agne.ovcinikova@gmail.com, \\ ${ }^{6}$ rasa.kizlaitiene@santa.lt, ${ }^{7}$ ieva.sereike@santa.lt, ${ }^{8}$ gintaras.kaubrys@santa.lt, ${ }^{9}$ j.pauk@pb.edu.pl, \\ ${ }^{10}$ a.idzkowski@pb.edu.pl
}

(Received 21 June 2016; accepted 23 June 2016)

\begin{abstract}
Multiple sclerosis (MS) is the most frequent neurological disease causing permanent disability in young adults. Subtle walking difficulties, such as reduced walking speed, step length, cadence and increased step width can be detected at an early stage of the disease. Main goal of this research is by using non-invasive wireless inertial sensors measure gait of MS patients in clinical setting and extract temporal biomechanical parameters that would allow objectively evaluate level of disability in MS patients. Analysis of 25-Foot walk showed that the duration of stance phase is approximately 1.6 times greater in MS group than in healthy control group, while the duration of swing phase in MS group is 1.3 times longer. In general, the MS patients are walking approximately 1.6 times slower.
\end{abstract}

Keywords: multiple sclerosis, gait, inertial sensors, biomechanics.

\section{Introduction}

Multiple sclerosis is the most frequent neurological disease causing permanent disability in young adults [1]. However, the cause of MS remains unknown. The disease onset is often polysymptomatic. Common symptoms and signs of MS include sensory symptoms in limbs or face, visual loss, acute or subacute motor weakness, diplopia, gait disturbance and balance problems, vertigo, bladder problems, acute transverse myelitis and pain. People with MS frequently have neuromuscular deficits such as ataxia, early muscle fatigue, spasticity and sensory disturbances, which limit gait and considerably affect their everyday living activities [1]. Subtle walking difficulties, such as reduced walking speed, step length, cadence and increased step width can be detected at an early stage of the disease [2, 3, 4, 5]. Walking limitations have negative consequences in activities of daily living, quality of life, as well as employment etc. Like in many neurological disorders, such evaluations are typically approached by direct observation of the clinician supported by a timed analysis, functional scales and questionnaires. Information derived from neurological assessment is included in the expanded disability status scale (EDSS) and it is most widely used to evaluate disability in MS, in both daily clinical practice and trials $[2,3,5]$. However, it is essential to find new tools, complementary to the clinical scales, able to supply objective and quantitative data useful in supporting clinical assessment of the disability as well as its variations across time. In addition, it is very important to have available reliable and accurate techniques to assess the degree of deviation from a physiological gait pattern as well as to detect even small changes in it consequent to pharmacological or rehabilitative treatment. Many methods have been reported in the last decade objectively acquire quantitative data on gait alterations of MS patients. Quantitative gait observation has been collected using several of techniques: video

(C) 2016 The Authors. Published by VGTU Press. This is an open-access article distributed under the terms of the Creative Commons Attribution License (CC-BY 4.0), which permits unrestricted use, distribution, and reproduction in any medium, provided the original author and source are credited. 
analysis, accelerometers [6,7] and three-dimensional motion capture systems [8]. However specialized motion-analysis laboratories were required to capture sophisticated gait and balance data [6]. Portable technologies now that collect equivalent data rapidly and with instant analysis are being widely explored for use in MS. These include accelerometers, pedometers, and pressure mats for gait analysis [6].

Main goal of this research is by using non-invasive wireless inertial sensors measure gait of multiple sclerosis patients in clinical setting and extract temporal biomechanical parameters that would allow objectively evaluate level of disability in MS patients.

\section{Methods}

Research was carried out on volunteers who were divided into two groups -11 control (CO) subjects ( 5 men, 6 women, aged: $31.5 \pm 4.7$ (mean \pm SD)), 14 multiple sclerosis (MS) subjects (6 men, 8 women, aged: $38.5 \pm 12.49$ (mean \pm SD)). None of the participants had any other injuries or disease affecting movement or coordination other than MS. All fourteen patients suffering from relapsing-remitting MS with EDSS score of $\leq 6$ (range 2.5-6, mean EDSS 4.1 \pm 1.4 (mean \pm SD)). The main inclusion criteria - the ability to walk independently without any assisting devices (i.e. crutches, working frames, foot supports and similar). Everyone provided informed consent prior to participating in the study. Neurological disability (EDSS score) was evaluated for each patient by a neurologist expert in MS.

Six, wireless inertial sensors (Shimmer Research, Dublin, Ireland), each able to measure linear acceleration, angular velocity and magnetic heading in three dimensions were attached to each patient's right and left thigh, shank and foot. The data from the sensors acquired via Bluetooth wireless connection at a sampling frequency of $51.2 \mathrm{~Hz}$ and stored on the computer for later processing. Each subject performed timed 25-Foot Walk (T25-FW) gait task at their selected pace as fast as possible. Each measurement was performed three times.

Following temporal parameters of gait have been selected for the analysis: stance phase duration (s), swing phase duration (s), average step time (s), approximate time of the whole gait task $(\mathrm{s})$, velocity $(\mathrm{m} / \mathrm{s})$, cadence $\left(\right.$ steps $\left.\times \mathrm{min}^{-1}\right)$, stride length $(\mathrm{m})$, coefficient of variability $(\mathrm{CV}, \%)$. Statistical analysis of the metrics was performed using IBM's SPSS v22 software. A one-way ANOVA with a significance level of $\alpha=0.05$ was used to test the null hypothesis that the means of gait parameters are the same between the MS and CO groups.

\section{Results}

The full set of metrics for the comparative analysis is presented in Table 1.

Table 1. Temporal gait parameters

\begin{tabular}{|c|c|c|c|c|}
\hline Parameters & Groups & Mean & SD & CV, \% \\
\hline \multirow{2}{*}{ Stance phase time, $\mathrm{s}$} & CO & 0.419 & 0.07 & 17.4 \\
& MS & 0.662 & 0.134 & 12.7 \\
\hline \multirow{2}{*}{ Swing phase time, s } & CO & 0.404 & 0.08 & 20.9 \\
& MS & 0.542 & 0.087 & 20.2 \\
\hline \multirow{2}{*}{ Step time, s } & CO & 0.824 & 0.135 & 16.4 \\
& MS & 1.204 & 0.153 & 16.2 \\
\hline \multirow{2}{*}{ Approximate motion time, s } & CO & 6.909 & 0.792 & 11.5 \\
& MS & 11.142 & 1.597 & 14.3 \\
\hline \multirow{2}{*}{ Cadence, steps $\times$ min $^{-1}$} & CO & 94.74 & 13.811 & 14.6 \\
& MS & 72.351 & 11.456 & 15.8 \\
\hline \multirow{2}{*}{ Stride length, m } & CO & 1.409 & 0.189 & 13.4 \\
& MS & 1.164 & 0.217 & 18.6 \\
\hline \multirow{2}{*}{ Velocity, $\mathrm{m} \times \mathrm{s}^{-1}$} & CO & 1.099 & 0.125 & 11.4 \\
& MS & 0.686 & 0.094 & 13.7 \\
\hline
\end{tabular}


A one-way between subjects ANOVA was conducted to compare the means of the gait metrics for analysis of MS and CO groups. When analysing T25-FW gait task, we found that there was a statistically significant difference in duration of the stance phase [ $F=47.42, p=0.0002]$, duration of the swing phase $[F=25.88, p=0.0008]$, step time $[F=66.39, p=0.0003]$, approximate motion time $[F=50.87, p=0.0006]$, cadence $[F=18.5, p=0.0003]$, stride length $[F=4.88, p=0.0391]$, velocity $[F=65.51, p=0.0009]$.

\section{Conclusions}

Analysis of 25-Foot walk showed that the duration of stance phase is approximately 1.6 times greater in MS group than in CO group, while the duration of swing phase in MS group is 1.3 times longer than in $\mathrm{CO}$ group. However, the coefficient of variability is greater in $\mathrm{CO}$ group during the stance phase time than indicating that there is some degree of inconsistency among the $\mathrm{CO}$ subjects, while the coefficient of variability of the swing phase duration is almost the same in both groups. Similarly, the variability of step time is the same in both groups, but in general, the MS patients are walking approximately 1.6 slower.

\section{References}

[1] Pau, M., et al. 2014. Novel characterization of gait impairments in people with multiple sclerosis by means of the gait profile score, Journal of Neurological Sciences 345(1-2): 159-163.

http://dx.doi.org/10.1016/j.jns.2014.07.032

[2] Pilutti, L. A., et al. 2013. Gait and six-minute walk performance in persons with multiple sclerosis, Journal of Neurological Sciences 334(1-2): 72-76. http://dx.doi.org/10.1016/j.jns.2013.07.2511

[3] Spain, R. I., et al. 2014. Body-worn sensors capture variability, but not decline, of gait and balance measures in multiple sclerosis over 18 months, Gait \& Posture 39(3): 958-964.

http://dx.doi.org/10.1016/j.gaitpost.2013.12.010

[4] Roeing, K. L., et al. 2015. Gait termination in individuals with multiple sclerosis, Gait \& Posture 42(3): 335-339. http://dx.doi.org/10.1016/j.gaitpost.2015.06.192

[5] Kalron, A. 2016. Gait variability across the disability spectrum in people with multiple sclerosis, Journal of Neurological Sciences 361: 1-6. http://dx.doi.org/10.1016/j.jns.2015.12.012

[6] Monticone, M., et al. 2014. Reliability of spatial-temporal gait parameters during dual-task interference in people with multiple sclerosis. A cross-sectional study, Gait \& Posture 40(4): 715-718. http://dx.doi.org/10.1016/j.gaitpost.2014.06.015

[7] Kotiadis, D.; Hermens, H. J.; Veltink, P. H. 2010. Inertial gait phase detection for control of a drop foot stimulator inertial sensing for gait phase detection, Medical Engineering \& Physics 32(4): 287297. http://dx.doi.org/10.1016/j.medengphy.2009.10.014

[8] Rueterbories, J., et al. Methods for gait event detection and analysis in ambulatory systems, Medical Engineering \& Physics 32(6): 545-552. http://dx.doi.org/10.1016/j.medengphy.2010.03.007 